\title{
Myocardial computed tomography perfusion
}

\author{
Kelley R. Branch ${ }^{1}$, Ryan D. Haley ${ }^{2}$, Marcio Sommer Bittencourt ${ }^{3,4}$, Amit R. Patel ${ }^{5}$, Edward Hulten ${ }^{2}$, Ron \\ Blankstein $^{6}$
}

${ }^{1}$ Department of Medicine (Cardiovascular Division), University of Washington, Seattle, WA, USA; ${ }^{2}$ Cardiology Service, Department of Internal Medicine, Walter Reed National Military Medical Center, Bethesda, MD, USA; ${ }^{3}$ Division of Internal Medicine, University Hospital and State of São Paulo Cancer Institute (ICESP), University of São Paulo, São Paulo, Brazil; ${ }^{4}$ Preventive Medicine Center Hospital Israelita Albert Einstein and School of Medicine, Faculdade Israelita de Ciência da Saúde Albert Einstein, São Paulo, Brazil; ${ }^{5}$ Department of Medicine, University of Chicago, Chicago, IL, USA; ${ }^{6}$ Cardiovascular Imaging Program, Departments of Medicine (Cardiovascular Division) and Radiology, Brigham and Women's Hospital, Harvard Medical School, Boston, MA, USA

Contributions: (I) Conception and design: KR Branch, R Blankstein; (II) Administrative support: KR Branch, R Blankstein; (III) Provision of study material or patients: None; (IV) Collection and assembly of data: All authors; (V) Data analysis and interpretation: All authors; (VI) Manuscript writing: All authors; (VII) Final approval of manuscript: All authors.

Correspondence to: Ron Blankstein, MD. Cardiovascular Imaging Program, Departments of Medicine (Cardiovascular Division) and Radiology, Brigham and Women's Hospital, 75 Francis St, Boston, MA 02115, USA. Email: rblankstein@partners.org.

\begin{abstract}
Despite having excellent diagnostic accuracy to detect anatomical coronary stenosis, coronary CT angiography (CTA) has a limited specificity to detect myocardial ischemia. CT perfusion (CTP) can identify myocardial perfusion defects during vasodilator stress, and when added to coronary CTA, improves the specificity of detecting hemodynamically significant stenosis. A CTP protocol typically involves the acquisition of two separate data sets: (I) a rest scan that can be used as both a coronary CTA and for evaluating rest myocardial perfusion, and (II) a stress CTP scan acquired during vasodilator stress testing. This review summarizes some the techniques, strengths, and limitations of CTP, and provides an overview of the recent evidence supporting the potential use of CTP in clinical practice.
\end{abstract}

Keywords: Stress test; CT perfusion (CTP); cardiac CT; myocardial perfusion imaging; ischemia

Submitted May 04, 2017. Accepted for publication Jun 20, 2017.

doi: $10.21037 / \mathrm{cdt} .2017 .06 .11$

View this article at: http://dx.doi.org/10.21037/cdt.2017.06.11

\section{Introduction}

Coronary computed tomography angiography (CTA) has a high sensitivity to rule out obstructive coronary artery disease $(\mathrm{CAD})$, though its specificity to identify myocardial ischemia is only modest, mostly due to the lower positive predictive value of obstructive lesions detected by coronary CTA (1). The lower specificity of CTA has contributed to a higher rate of downstream invasive coronary angiography (ICA) and coronary revascularization in patients undergoing coronary CTA versus functional testing, despite uncertain clinical benefit of such procedures (2-4). Although recent studies suggest coronary CTA may result in improved outcomes, the overall ICA and revascularizations rates remains higher than desired $(4,5)$.
Addressing these limitations, several advances in cardiac CT have permitted adding functional information to the routinely acquired anatomical data. Among those techniques is the development of CT perfusion (CTP) imaging. This technique has been shown to improve the specificity and overall accuracy of cardiac CT to detect flow limiting CAD or areas of prior myocardial infarction (6-9). The evaluation of myocardial ischemia is particularly important in situations where the anatomical information might be inconclusive, such as in patients with moderate coronary stenosis. This manuscript will review the current status of CT myocardial perfusion as well as outline future refinements and research pathways necessary for clinical adoption of this technology. 
Table 1 Summary of advantages and disadvantages of CT perfusion

\begin{tabular}{lll}
\hline Categories & Advantages & Disadvantages \\
\hline Static CTP & CT coronary angiography and CT perfusion with single data set & Qualitative and semi-quantitative CTP analyses only \\
& Rapid analysis & Susceptible to artifacts \\
& Less dependent on specific vendor technology & Timing of acquisition essential \\
& Most data on diagnostic accuracy and outcomes & \\
& Lower radiation dose & Long breath hold \\
Dynamic CTP & $\begin{array}{l}\text { Qualitative, semi- and fully quantitative (mL/min/gram) } \\
\text { measurements possible } \\
\end{array}$ & \\
& Ability to observe first pass of myocardial perfusion, which may mitigate some artifacts & More susceptible to patient and cardiac motion artifacts \\
& Timing less critical than static CTP & Higher radiation dose \\
& & $\begin{array}{l}\text { Increased image noise (due to multiple data sets being } \\
\text { acquired) }\end{array}$ \\
& Longer and more complex analysis
\end{tabular}

CTP, CT perfusion

\section{Basic CTP concepts and acquisition methods}

Myocardial CTP is in many ways similar to nuclear and magnetic resonance perfusion imaging techniques. During CTP, radiopaque iodinated contrast is injected intravenously, transits the cardiac and pulmonary structures and then perfuses through the coronary vasculature and myocardium. This "first pass" of contrast through the myocardial vasculature is directly proportional myocardial blood flow. In turn, myocardial blood flow can be measured by two different techniques (Table 1). On static perfusion imaging, one single image may be used to compare myocardial CTP from one region of the myocardium to other remote areas. Alternatively, several sequential images can be acquired over time to determine both contrast perfusion into the myocardium as well as wash out. This technique is referred to as dynamic perfusion, and allows for semi-quantitative and fully quantitative assessment of myocardial blood flow.

A full CTP protocol requires the acquisition of both rest and stress images (Figure 1). Stress CTP is performed using vasodilator pharmacologic stress agents, not physical stress or dobutamine. Similar to late gadolinium enhancement imaging by cardiac magnetic resonance imaging, areas of scar have delayed washout of contrast out of the interstitial space. Thus, late enhancement of retained iodinated contrast can be used to detect fibrosis and myocardial scar. However, this requires a third acquisition approximately
5-10 minutes after the last contrast injection and is usually performed only in selected cases where such information is clinically needed. The combination of rest, stress and/or late enhancement perfusion images allows the differentiation of normal myocardial flow from myocardial ischemia and infarction. To date, various techniques for CTP acquisition and analysis have been reported and there currently is no consensus on CTP performance, since each protocol, technique, and analysis have advantages and disadvantages as considered below.

\section{Static CTP}

Static CTP is a single imaging set acquired similarly to coronary CTA. Static CTP is timed at or just after the peak of myocardial contrast opacification where separation of contrast content between normal, ischemic and infarcted tissue is highest. This has the advantage of acquiring both coronary CTA and CTP simultaneously and with a single contrast bolus. The exact techniques for CTP vary depending on the CT scanner used although CTP settings and imaging techniques are similar to the typical settings used for acquiring coronary CTA. Virtually any $\mathrm{CT}$ acquisition technique can be used including retrospectively ECG-gated helical scanning, prospective ECG-triggered scanning or high-speed helical scanning. Static CTP generally requires $50-80 \mathrm{~mL}$ of iodinated 


\section{Cardiac CT Myocardial Perfusion}
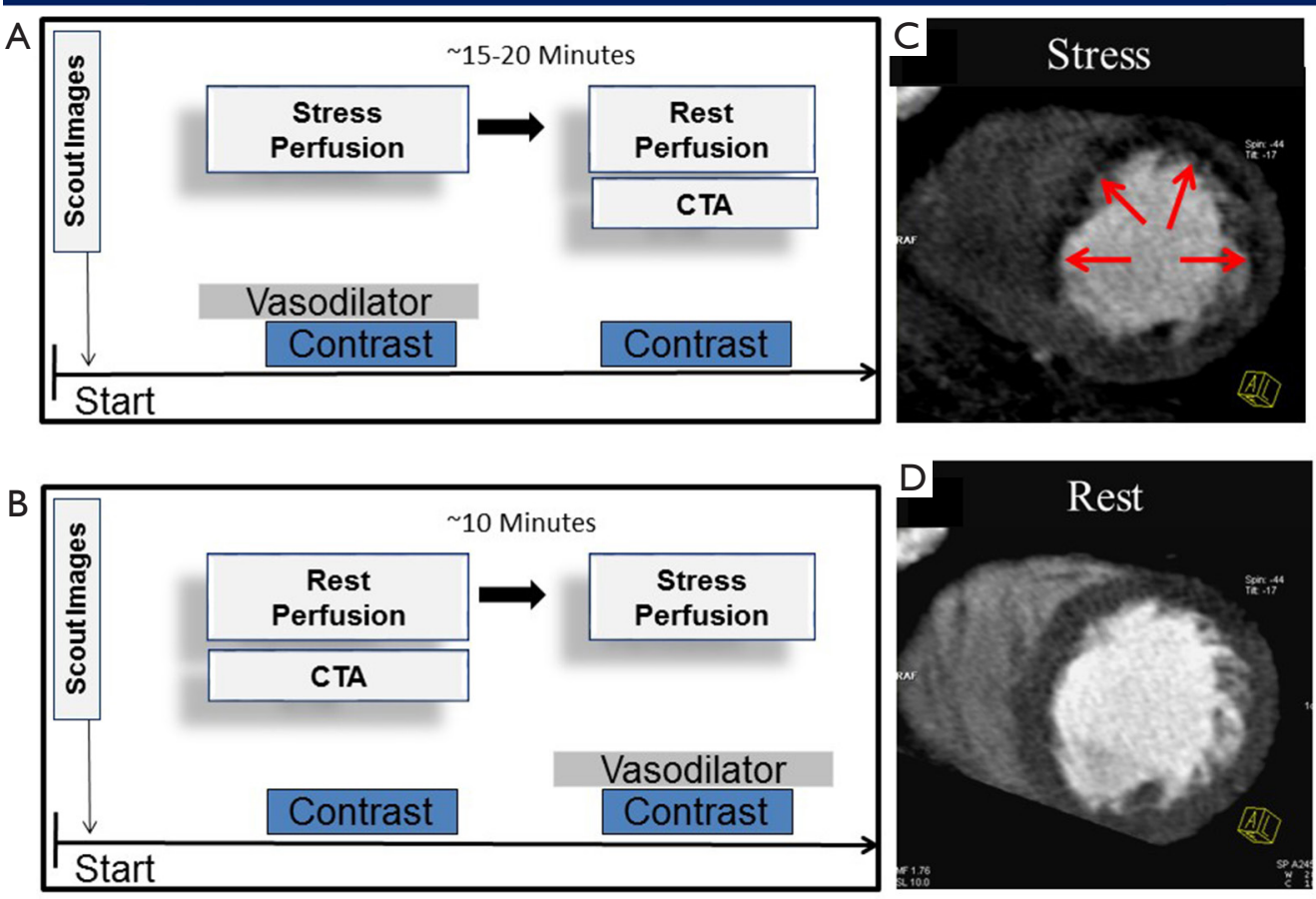

Figure 1 Example of two possible CT perfusion protocols. (A) stress first protocol—stress perfusion is completed first followed by a delay of 15-20 minutes and then rest perfusion and coronary CTA; (B) rest first protocol—rest perfusion and coronary CTA is completed first followed by stress perfusion; (C) the images on the right show stress CTP image which shows a perfusion defect throughout the anteroseptal, anterior, and anterolateral wall; (D) the same patient had a normal rest myocardial perfusion image with no defects. CTA, CT angiography; CTP, CT perfusion.

contrast bolus injected intravenously at $4-6 \mathrm{~mL} / \mathrm{second}$ followed by a saline bolus. To determine correct timing of the CT scan, real time bolus tracking of aortic contrast enhancement or a test bolus of 10-20 mL can be used to determine the ideal time necessary to achieve peak contrast opacification in the myocardium, typically 4 seconds after contrast peaks in the ascending aorta (10). Scanner settings include a variable $\mathrm{mA}$ depending on body habitus and a $\mathrm{kVp}$ of $80-120 \mathrm{mV}$, though lower $\mathrm{kVp}$ is preferred for better contrast to noise ratio in CTP as well as for lower radiation exposure for the patient. With wide bore detectors that cover the entire $\mathrm{Z}$-axis of the heart, coronary CTA and CTP are timed to ensure that contrast has reached all portions of the myocardium, and the acquisition is performed using a single data set and without any table motion. In scanners that have smaller $\mathrm{Z}$-axis coverage, the coronaries and myocardium are imaged over time and a single data set often incorporates data from multiple table positions, using either a helical mode or an axial mode. In this case, the acquisition timing is even more important, as inaccurate timing may worsen step artifacts through the myocardium, which may lead to incorrect interpretation of perfusion abnormalities (11).

\section{Dynamic CT}

In contrast to single image static CT, dynamic CT acquires multiple CT images as contrast moves through the myocardium (Figure 2). Because of the need for repeated myocardial imaging, dynamic CT is generally performed with wide bore or high helical pitch scanners where the latter uses repeated shuffling of patients in and out of the scanner to cover the cardiac z-axis. To ensure correct acquisition of myocardial enhancement over time, an initial timing bolus is commonly used to time the dynamic CT scan to start 4 to 6 seconds prior to contrast arrival at the left ventricle or ascending aorta. CT acquisitions then occur every 1-3 seconds for the next 20 to 40 seconds. This has 


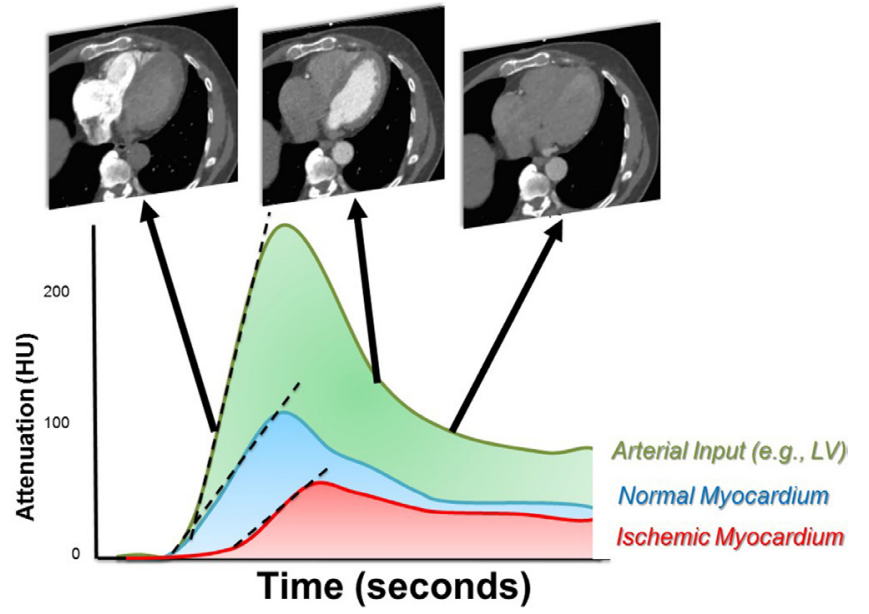

Figure 2 Time attenuation curves for normal myocardium (blue), ischemic myocardium (red) and the arterial input function (AIF; green). Representative CT images for certain time points are shown above the graph. The upslopes are represented by the dashed lines and are used to calculate semiquantitative myocardial blood flow. HU, Hounsfield units; LV, left ventricle.

the distinct advantage of imaging the entire "first pass" of contrast through the myocardium like dynamic MRI. In this mode, the myocardial blood flow may be semi- or fully quantitatively measured. However, this acquisition mode has some challenges including patient discomfort and possible motion artifacts due to the required long breath hold. In addition, the repeated scans result in a significantly higher radiation exposure. This can be compensated with the use of low radiation dose images, though this may result in noisier images.

\section{Dual energy $C T$}

Various chemical compounds within cardiac tissue react differently to the spectra of CT photon energies and certain $\mathrm{keV}$ spectra have attenuation spikes (termed the $\mathrm{K}$ spike) that can improve the image signal to noise ratio. While CTP acquisition is similar to other techniques, differing energies can be delivered by either two orthogonal CT tubes with a dual source gantry, by rapid $\mathrm{kVp}$ switching during CT gantry rotation or alternating $\mathrm{kVp}$ with each rotation, by filtering the photon energies using different metallic filters, or by delineating different photon energies with layered detectors. Dual or spectral energy CT has the potential to differentiate iodinated contrast from other cardiac compounds, like water and calcium, and thus has applicability to CTP. Despite these potential advantages, data showing the incremental benefit of dual energy CT beyond standard CTA techniques is lacking.

\section{Stress CTP imaging}

Stress CTP imaging uses vasodilator stress agents such as regadenoson, adenosine, and dipyridamole to evaluate for ischemia. The protocols are similar to those used during other non-invasive stress tests and these agents have been reviewed extensively elsewhere (12). However, for continuous infusion agents, such as adenosine or dipyridamole, two IV accesses are required, one for contrast and one for stress agents, as opposed to only requiring a single IV for both the single regadenoson bolus and IV contrast. Regardless of the agents used, heart rates increase approximately 10-20 bpm that may make coronary and myocardial imaging more challenging $(6,13)$. In addition, patients commonly have drug side effects such as dyspnea, chest pain, or nausea (12) which may create patient discomfort and motion especially with the long breath holds with dynamic CT.

\section{CTP imaging protocols}

Most CTP protocols incorporate a resting scan, which is also used for evaluating the coronary analysis, a stress scan, and in selected cases, a late enhancement scan. A common protocol has resting CTP with coronary CTA first followed by stress CT. A compelling reason for this imaging order is that a normal coronary CTA may allow deferment of stress CT imaging. In clinical practice, however, rapid coronary CTA processing may not always be possible. Another limitation of the rest first technique is that some contrast may remain in areas of scar, and thus result in a false negative finding on the subsequent stress CTP scan. An alternative protocol is stress CT imaging first followed by resting CTP with coronary CTA. This protocol could obviate the need for rest CT scanning if the stress perfusion images do not show any defects and the coronary arteries have no stenosis. However, because motion artifacts are common during vasodilator stress testing, it is likely that a rest CTA would still be required in many cases.

To date, most protocols perform resting CT followed by stress CT. However, in patients that have known obstructive $\mathrm{CAD}$ or those who have a large amount of coronary artery calcifications, we suggest performing a stress-first protocol (Figure 3), as in such cases it is likely that both stress and 


\begin{tabular}{|l|c|c|}
\hline Parameter & $\begin{array}{c}\text { Favor Stress } \\
\text { First }\end{array}$ & $\begin{array}{c}\text { Favor Rest } \\
\text { First }\end{array}$ \\
\hline $\begin{array}{l}\text { Low to intermediate pre-test } \\
\text { probability of CAD }\end{array}$ & $\mathrm{X}$ \\
\hline $\begin{array}{l}\text { Severe amount of coronary } \\
\text { artery calcifications }\end{array}$ & $\mathrm{X}$ \\
\hline Known prior CAD / PCI & $\mathrm{X}$ \\
\hline Prior MI & \\
\hline
\end{tabular}

Figure 3 Features that may be used in deciding whether to perform a stress-first or rest-first acquisition.

rest images would be required.

Importantly, to ensure clearance of circulating contrast, and to ensure that the pharmacological effects of longer acting stress agents regadenoson or dipyridamole are no longer present following a stress first approach, it is recommended to wait at least 15-20 minutes between scans. This this interval could be shortened if the reversal agent aminophylline is administered (1).

\section{CT myocardial perfusion analysis}

Post-processing of static CTP images is similar to nuclear imaging and generally analyzed visually where a myocardial segment with comparatively "normal" blood flow is required to visually detect segments with lower blood flow. Multiplanar reconstructions place the myocardial structures into orthogonal views as well as a short axis stack of images to allow analysis using the standardized 17 segment model. For static perfusion, various reconstruction techniques have been described for optimal visualization of hypoperfusion. In order to account for image noise, it is suggested to use appropriate image display settings that optimize visualization of the myocardium, including thick MPR slabs (6-8 $\mathrm{mm}$ ) using an average or minimum intensity projection (MinIP) rather than maximum intensity projections (MIP; Figure 4) $(6,13,14)$. CTP images should be viewed with a narrow window and level of approximately 300 and 100, respectively, which is more attuned to the Hounsfield units (HU) of myocardial contrast enhancement (15). Other suggested techniques include additional image processing tools such as smoothing filters or beam hardening artifact corrections when available.

Static CTP images are commonly visualized using a grayscale display, and qualitatively described as mild, moderate, or severe perfusion defect (Figure 5). For each defect, the location and reversibility should also be described. Similar to nuclear myocardial perfusion imaging, regional wall motions abnormalities during peak stress may be used to identify abnormal blood flow (16). However, a complete multiphase data set is often not acquired to avoid excess radiation exposure. However, all available phases should be evaluated for perfusion defects, as this can help distinguish artifacts (which may only be present on one phase) from true perfusion defects (which typically persist on multiple phases).

To further enhance CTP specificity, some investigators have applied a HU color map to the images, compared CTP to a normal cohort, or normalized to an average myocardial HU (Figure 4) (11,17). In addition, semi-quantitative perfusion measurements can also be performed using actual HU with normal myocardial perfusion averaging 90-100 HU (18) with ischemia and infarction ranging from negative HU to a mean of approximately $30 \mathrm{HU}$ (19). The trans-myocardial perfusion ratio (TPR) measures the ratio of the average subendocardial $H U$ to subepicardial HU where normal TPR is greater than one (20). A TPR below 0.75 is suggestive of ischemia and has even been used to differentiate normal myocardium from balanced ischemia (20). Interestingly, visual analysis appears to be more accurate overall and thus semiquantitative TPR evaluation remains an adjunct to qualitative analyses. At present, relative superiority of each of these techniques remains an important but largely unresolved question, and clinical reports are mostly based on subjective image analysis.

A significant limitation to both qualitative and quantitative CTP analyses is imaging artifacts (Figure 6). The most common artifact is beam hardening which generally occurs at the inferior base of the myocardium due to interpositioning of the highly attenuating spine and contrast filled descending aorta between the CT tube and myocardium $(21,22)$. This essentially creates "photon starvation" at the inferior base and manifests as an area of hypoperfusion. Various imaging-based and raw data-based algorithms have been proposed to obviate this artifact (23), although few are currently in routine use. Another important artifact is from cardiac motion, especially as 


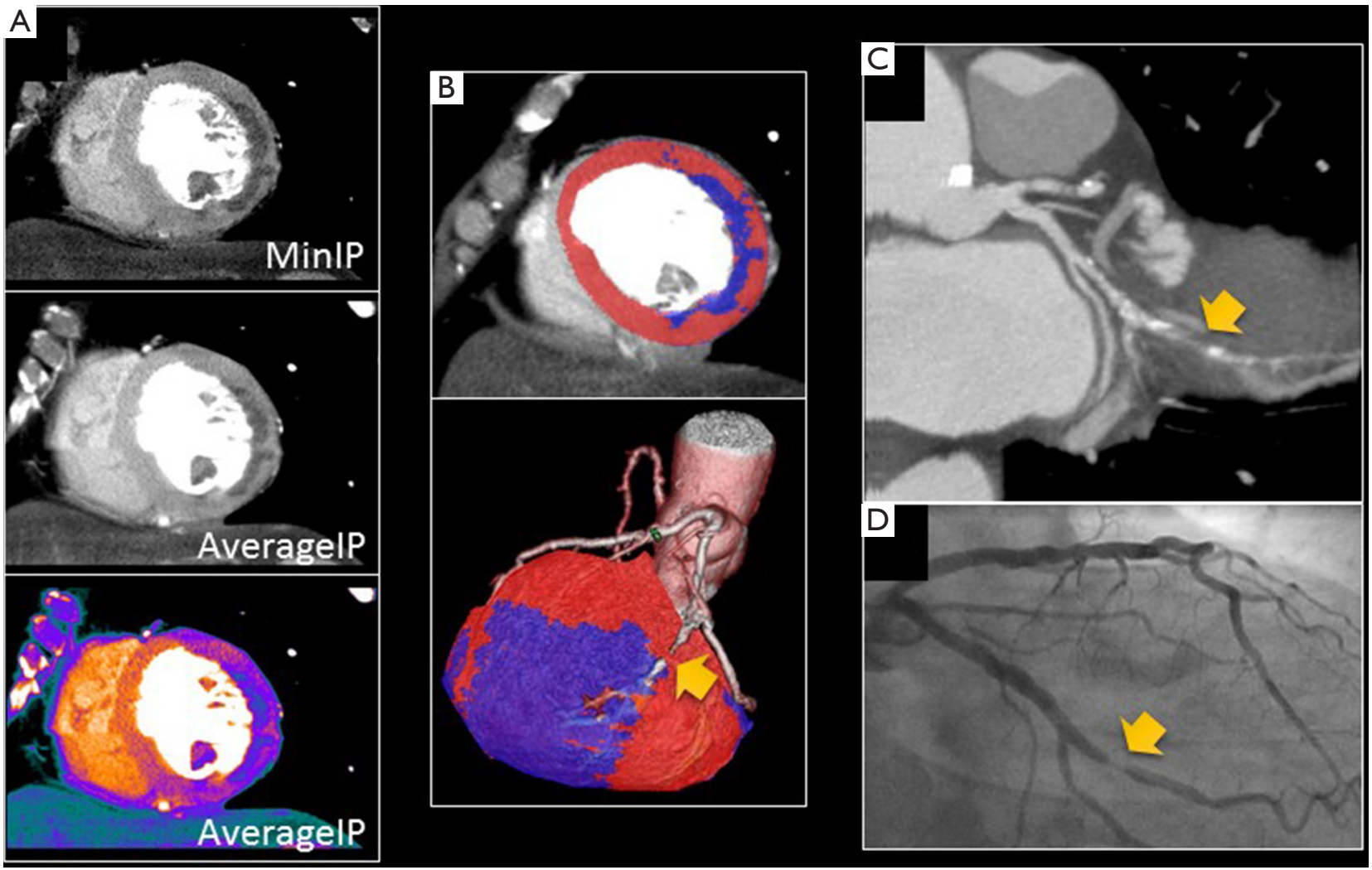

Figure 4 CT perfusion reconstructions in a patient with lateral wall infarction with (A) minimum intensity projection (MinIP) and average intensity projection grayscale and color HU map; (B) enhanced voxel distribution where $>1$ SD from the average HU of the myocardium shows blue suggesting hypoattenuation. High grade obtuse marginal stenosis (arrows) in the CT angiogram (C) and invasive coronary angiogram (D).

vasodilator medications can cause a significant increase in heart rate. Beta blockers are generally avoided during stress CTP due to concerns of affecting ischemia identification, although they can be given if the rest images are performed first, or after stress perfusion is completed. In addition, small z-axis detectors can have other challenges of banding artifacts, which are generally caused by myocardial motion within a detector coverage zone, as well variable contrast content from the superior to inferior myocardium due to contrast wash out during the longer acquisition required to cover the heart (Figure 6).

For dynamic CTP, qualitative, semi-quantitative and quantitative measurements are possible. Post-processing for visual analysis is similar to static CT although it is performed on all images through time. These images may be evaluated for relative areas of hypoperfusion over time similar to dynamic MRI. However, the theoretic benefit of dynamic CT is more robust semi and fully quantitative myocardial blood flow analyses. The relative enhancement of each pixel in the myocardium and cardiac cavities is measured over time to generate time attenuation curves (TAC's; Figure 2) for both the myocardium and arterial input (taken from the left atrium or ventricle). The myocardial TAC's can then be amalgamated into myocardial segments or total myocardial blood flow for mathematical analysis. In areas of impaired blood flow due to ischemia or infarction, the peak enhancement, rate of rise of myocardial enhancement, and the area under the TAC curve are diminished. To account for differences in contrast delivery and cardiac output, the arterial input function is used as a normalization factor. Since the CT attenuation in HU has a linear correlation with the iodine concentration, the myocardial upslope to left ventricular upslope are strongly associated with myocardial blood flow in animal models (24). Using a model-based deconvolution analysis, the absolute myocardial blood flow ( $\mathrm{mL} / \mathrm{min} / \mathrm{gram}$ of myocardial tissue) 


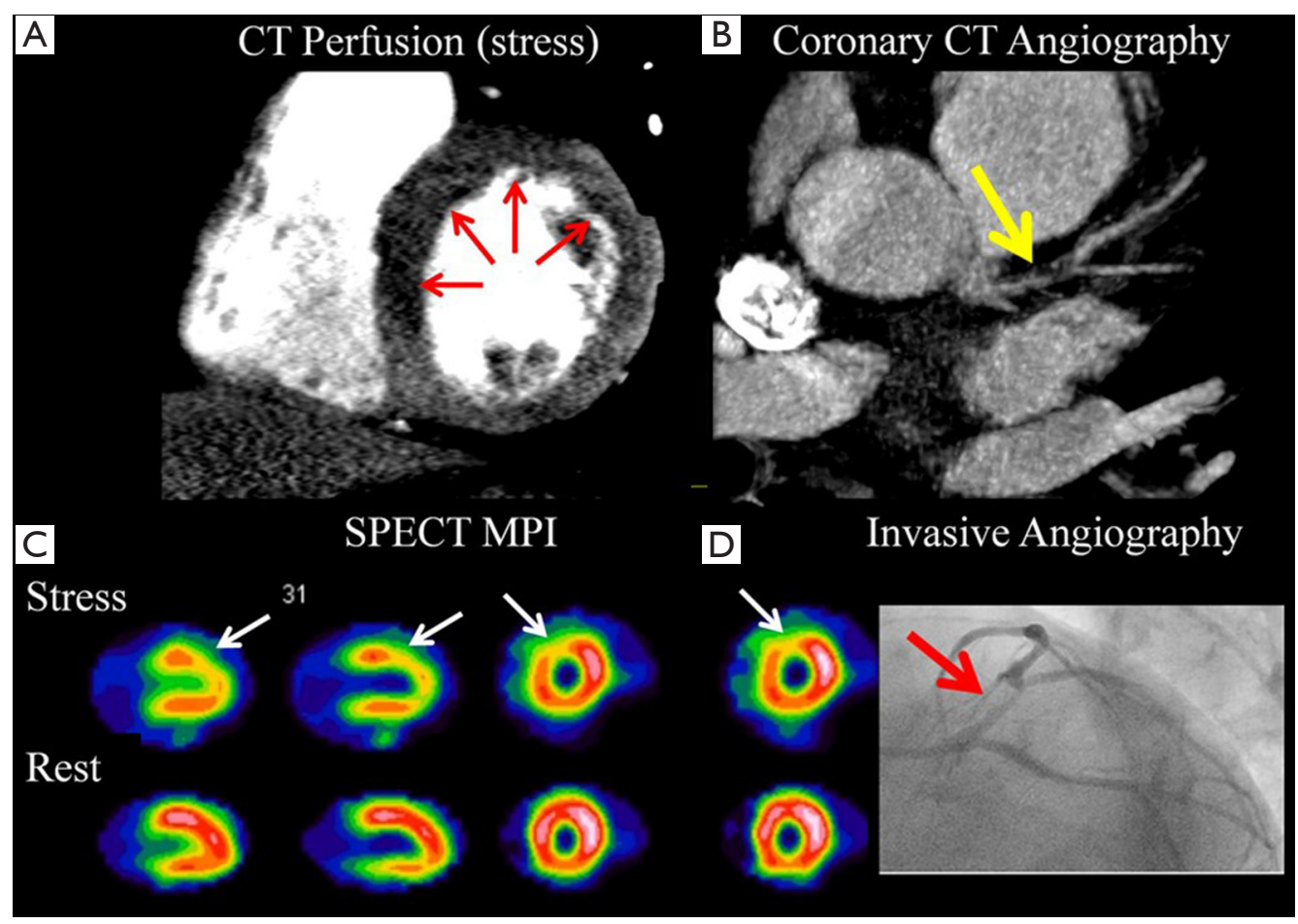

Figure 5 A 59-year-old obese man with no prior cardiac history who presented with chest pain and dyspnea on exertion. (A) A CT perfusion showed a large, severe perfusion defect in the anteroseptal, anterior, and anterolateral walls; (B) the CT angiography showed large noncalcified plaque in the proximal LAD; (C) SPECT MPI images with fully reversible defect throughout the mid and apical anterior wall; (D) invasive angiography in the left anterior oblique caudal view showing severe stenosis in LAD before the takeoff of the first diagonal branch. Reproduced from J Am Coll Cardiol (6) with permission from Elsevier.

can be estimated based on the dynamic CT myocardial attenuation values $(24,25)$. Quantitative modeling techniques are mathematically complex but in simple terms, account for flow into the myocardial vasculature, the transport kinetics for the relatively slow extravasation of contrast into the interstitium, and myocardial mass. Although other variables that may affect quantitation have been considered, the judicious two compartment models (with one compartment as myocardial vasculature and the other, the myocardial interstitium) approximate flow nearly as well as other or more complex models (26). More recent studies have also demonstrated that the CT derived MBF measurement has an excellent correlation with invasive fractional flow reserve (FFR) (1) and with MBF measured by positron emission tomography (27).

\section{Diagnostic accuracy of CT myocardial perfusion}

Despite numerous validations studies for both static and dynamic CT myocardial perfusion studies, the diagnostic accuracy of this technology is still debated. This is due, in part to the high variability in acquisition techniques used by various studies, as well as the large number of different reference standards including nuclear single photon emission computed tomography (SPECT) perfusion studies $(28,29)$, magnetic resonance stress perfusion (30), positron emission tomography (PET) (27), invasive angiography and invasive FFR $(31,32)$. One meta-analysis has evaluated the sensitivity and specificity of CT myocardial perfusion compared only to ICA and FFR and estimated a CTP sensitivity of $81 \%$ and a specificity of $93 \%$ (33). A more recent meta-analysis has stratified the accuracy of CT myocardial perfusion according the CT acquisition mode, using various modalities as the reference standards. Despite the limitation of combining heterogeneous reference standards, the overall sensitivity varied from $75 \%$ to $84 \%$, with specificities from $78 \%$ to $95 \%$ (34).

Besides the single center trials, two randomized trials have also evaluated the accuracy of CTP. The CORE320 study evaluated 381 individuals undergoing coronary CTA 

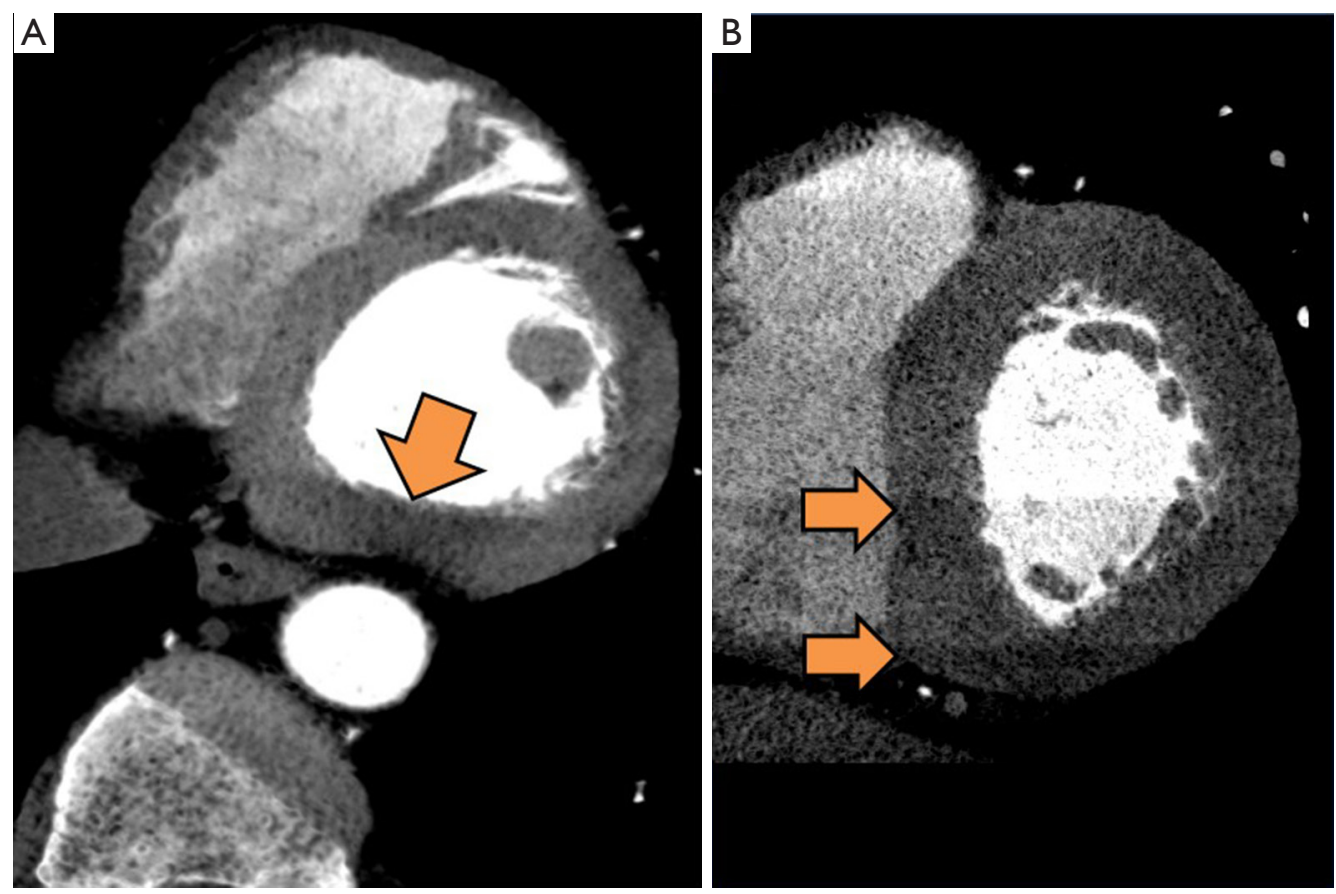

Figure 6 CT perfusion artifacts with (A) beam hardening (arrow) in basal inferolateral wall due to interposed spine and contrast filled aorta and (B) banding artifact (arrows) due to differences in contrast uniformity during a scan acquisition with smaller detector scanners that requires multiple heart beats.

plus CTP, in addition to invasive angiography and SPECT myocardial perfusion imaging. The combination of CTA and CTP had a sensitivity of $80 \%$ and specificity of $74 \%$, when compared to a reference standard of invasive angiography and SPECT MPI. Importantly, for both CTA and invasive angiography, lesions of $\geq 50 \%$ stenosis had to have a perfusion defects (whether by CT or SPECT MPI) in order to be categorized as abnormal (29). As expected, the specificity of any angiographic finding on CTA increased the more severe the perfusion defect on CTP, as quantified by the CTP stress summed score. The second multicenter trial by Cury et al. performed regadenoson rest/stress CT myocardial perfusion in 110 subjects using various CT scanners from 64 to 320 detectors and compared to SPECT imaging. CTP sensitivity was $90 \%$ and specificity was $84 \%$ and was deemed non-inferior to SPECT (28). Interestingly, this trial included multiple sites which did not have prior experience in performing CTP, suggesting that despite its technical nature, CTP can be successfully adopted by various sites.

Most studies to date have evaluated the independent accuracy of CT myocardial perfusion to detect obstructive $\mathrm{CAD}$ and/or myocardial ischemia. However, it is unlikely that CT myocardial perfusion will be used this way. Since the anatomical CTA information is already available, the contribution of CTP beyond the anatomical information is of most interest, except for situations where the coronary vessel is significantly limited by artifacts, heavy calcifications or the presence of stents (35). The CORE320 study demonstrated that the addition of static CTP to the coronary CTA improved the specificity from $51 \%$ to $74 \%$ but at the cost of a reduction in sensitivity (92\% to 80\%) (29). Despite these changes, the overall diagnostic accuracy measured by area under the receiver operator curve increased from 0.84 for CTA ( $\geq 50 \%$ stenosis) alone to 0.87 for combined CTACTP. Not surprisingly, combined CTA-CTP accuracy was less in patients with stenting, prior myocardial infarction or uninterpretable CTA. Other smaller studies have shown similar incremental changes in accuracy with the combining CTA and static CTP information (9).

\section{CTP limitations and future directions}

While there are limitations to the current CTP literature, significant advances continue, and the capabilities and performance of this technique are expected to further improve. As stated previously, significant heterogeneity 
of CTP analytic techniques within the literature affect comparisons between studies and there remains equipoise as to whether static or dynamic CTP are the optimal technique. Future studies should further define the most optimal CTP techniques, although these may in part be dependent on the scanner used. In addition, future studies should standardize how CTP can determine not just the presence or absence of ischemia, but also quantify the amount of ischemia, similar to the various semi-quantitative scores that are used in nuclear cardiology. From a technical perspective, future studies should determine how to mitigate various artifacts, ranging from motion to beam hardening.

While there are various imperfect "gold standard" comparators, we suggest that future studies employ techniques such as invasive angiography plus FFR, or positron emission tomography (ideally with quantitative coronary flow reserve), in order to provide a more valid reference standard. In addition, future outcomes data regarding CTP and how it may influence patient management are needed. To date, only one study from the CORE320 trial reported outcome data (36), and showed that 2-year major adverse cardiac events were similar for combined CTA-CTP as for invasive coronary angiography (ICA) and SPECT performed in the same subjects. However, further trials are needed to ensure that the theoretical benefits of combined anatomic and functional CTA-CTP analyses translate into improved resource utilization and improved clinical outcomes.

\section{Conclusions}

Despite the use of multiple protocols and various artifacts, the available data suggests that CTP may have an accuracy which is comparable to SPECT MPI for identifying myocardial ischemia. Ultimately, CTP may serve as a useful adjunct to coronary CTA to improve the specificity of detecting myocardial ischemia. Future advances in CTP will likely improve the diagnostic accuracy of CTP + CTA, and enable semi- or fully quantitative techniques that will better estimate the severity of ischemia.

\section{Acknowledgements}

None.

\section{Footnote}

Conflicts of Interest: The authors have no conflicts of interest to declare.

Disclaimer: The opinions and assertions contained herein are the authors' alone and do not reflect the views of Walter Reed National Military Medical Center, the US Army, or the Department of Defense.

\section{References}

1. Rossi A, Dharampal A, Wragg A, et al. Diagnostic performance of hyperaemic myocardial blood flow index obtained by dynamic computed tomography: does it predict functionally significant coronary lesions? Eur Heart J Cardiovasc Imaging 2014;15:85-94.

2. Shreibati JB, Baker LC, Hlatky MA. Association of coronary CT angiography or stress testing with subsequent utilization and spending among Medicare beneficiaries. JAMA 2011;306:2128-36.

3. Hulten E, Goehler A, Bittencourt MS, et al. Cost and resource utilization associated with use of computed tomography to evaluate chest pain in the emergency department. Circ Cardiovasc Qual Outcomes 2013;6:514-24.

4. Bittencourt MS, Hulten EA, Murthy VL, et al. Clinical outcomes after evaluation of stable chest pain by coronary computed tomographic angiography versus usual care: a meta-analysis. Circ Cardiovasc Imaging 2016;9:e004419.

5. Jørgensen ME, Andersson C, Nørgaard BL, et al. Functional testing or coronary computed tomography angiography in patients with stable coronary artery disease. J Am Coll Cardiol 2017;69:1761-70.

6. Blankstein R, Shturman LD, Rogers IS, et al. Adenosineinduced stress myocardial perfusion imaging using dualsource cardiac computed tomography. J Am Coll Cardiol 2009;54:1072-84.

7. Hulten EA, Bittencourt MS, Ghoshhajra B, et al. Stress CT perfusion: Coupling coronary anatomy with physiology. J Nucl Cardiol 2012;19:588-600.

8. George RT, Arbab-Zadeh A, Miller JM, et al. Adenosine stress 64- and 256-row detector computed tomography angiography and perfusion imaging: a pilot study evaluating the transmural extent of perfusion abnormalities to predict atherosclerosis causing myocardial ischemia. Circ Cardiovasc Imaging 2009;2:174-82.

9. Siontis KC, Gersh BJ, Williamson EE, et al. Diagnostic performance of myocardial CT perfusion imaging with or without coronary CT angiography. JACC Cardiovasc Imaging 2016;9:322-4. 
10. Vliegenthart R, Henzler T, Moscariello A, et al. CT of coronary heart disease: Part 1, CT of myocardial infarction, ischemia, and viability. AJR Am J Roentgenol 2012;198:531-47.

11. Busch JL, Alessio AM, Caldwell JH, et al. Myocardial hypo-enhancement on resting computed tomography angiography images accurately identifies myocardial hypoperfusion. J Cardiovasc Comput Tomogr 2011;5:412-20.

12. Henzlova MJ, Duvall WL, Einstein AJ, et al. ASNC imaging guidelines for SPECT nuclear cardiology procedures: Stress, protocols, and tracers. J Nucl Cardiol 2016;23:606-39.

13. Kurata A, Mochizuki T, Koyama Y, et al. Myocardial perfusion imaging using ademosine triphosphate stress milti-slice spiral computed tomography: Alternative to stress myocaridla perfusion scintography. Circ J 2005;69:550-7.

14. Rogers IS, Cury RC, Blankstein R, et al. Comparison of postprocessing techniques for the detection of perfusion defects by cardiac computed tomography in patients presenting with acute ST-segment elevation myocardial infarction. J Cardiovasc Comput Tomogr 2010;4:258-66.

15. Blankstein R, Rogers IS, Cury RC. Practical tips and tricks in cardiovascular computed tomography: diagnosis of myocardial infarction. J Cardiovasc Comput Tomogr 2009;3:104-11.

16. Cury RC, Nieman K, Shapiro MD, et al. Comprehensive assessment of myocardial perfusion defects, regional wall motion, and left ventricular function by using 64-section multidetector CT. Radiology 2008;248:466-75.

17. Kachenoura N, Gaspar T, Lodato JA, et al. Combined assessment of coronary anatomy and myocardial perfusion using multidetector computed tomography for the evaluation of coronary artery disease. Am J Cardiol 2009; 103:1487-94.

18. Stanton CL, Haramati LB, Berko NS, et al. Normal myocardial perfusion on 64-detector resting cardiac CT. J Cardiovasc Comput Tomogr 2011;5:52-60.

19. Nieman K, Cury RC, Ferencik M, et al. Differentiation of recent and chronic myocardial infarction by cardiac computed tomography. Am J Cardiol 2006;98:303-8.

20. Cury RC, Magalhaes TA, Paladino AT, et al. Dipyridamole stress and rest transmural myocardial perfusion ratio evaluation by 64 detector-row computed tomography. J Cardiovasc Comput Tomogr 2011;5:443-8.

21. Kitagawa K, George RT, Arbab-Zadeh A, et al. Characterization and correction of beam-hardening artifacts during dynamic volume CT assessment of myocardial perfusion. Radiology 2010;256:111-8.

22. Rodríguez-Granillo GA, Rosales MA, Degrossi E, et al. Signal density of left ventricular myocardial segments and impact of beam hardening artifact: implications for myocardial perfusion assessment by multidetector CT coronary angiography. Int J Cardiovasc Imaging 2010;26:345-54.

23. So A, Hsieh J, Li JY, et al. Beam hardening correction in CT myocardial perfusion measurement. Phys Med Biol 2009;54:3031-50.

24. George RT, Jerosch-Herold M, Silva C, et al. Quantification of myocardial perfusion using dynamic 64-detector computed tomography. Invest Radiol 2007;42:815-22.

25. Ho KT, Chua KC, Klotz E, et al. Stress and rest dynamic myocardial perfusion imaging by evaluation of complete time-attenuation curves with dual-source CT. JACC Cardiovasc Imaging 2010;3:811-20.

26. Meinel FG, Ebersberger U, Schoepf UJ, et al. Global quantification of left ventricular myocardial perfusion at dynamic CT: feasibility in a multicenter patient population. AJR Am J Roentgenol 2014;203:W174-80.

27. Kikuchi Y, Oyama-Manabe N, Naya M, et al. Quantification of myocardial blood flow using dynamic 320-row multi-detector CT as compared with 15O-H2O PET. Eur Radiol 2014;24:1547-56.

28. Cury RC, Kitt TM, Feaheny K, et al. A randomized, multicenter, multivendor study of myocardial perfusion imaging with regadenoson CT perfusion vs single photon emission CT. J Cardiovasc Comput Tomogr 2015;9:10312.e1-2.

29. Rochitte CE, George RT, Chen MY, et al. Computed tomography angiography and perfusion to assess coronary artery stenosis causing perfusion defects by single photon emission computed tomography: the CORE320 study. Eur Heart J 2014;35:1120-30.

30. Ko SM, Park JH, Hwang HK, et al. Direct comparison of stress- and rest-dual-energy computed tomography for detection of myocardial perfusion defect. Int J Cardiovasc Imaging 2014;30 Suppl 1:41-53.

31. Yang DH, Kim YH, Roh JH, et al. Stress myocardial perfusion ct in patients suspected of having coronary artery disease: visual and quantitative analysis-validation by using fractional flow reserve. Radiology 2015;276:715-23.

32. Ko BS, Cameron JD, Meredith IT, et al. Computed tomography stress myocardial perfusion imaging in patients considered for revascularization: a comparison 
with fractional flow reserve. Eur Heart J 2012;33:67-77.

33. Tashakkor AY, Nicolaou S, Leipsic J, et al. The emerging role of cardiac computed tomography for the assessment of coronary perfusion: a systematic review and meta-analysis. Can J Cardiol 2012;28:413-422.

34. Pelgrim GJ, Dorrius M, Xie X, et al. The dream of a onestop-shop: Meta-analysis on myocardial perfusion CT. Eur J Radiol 2015;84:2411-20.

35. Magalhães TA, Kishi S, George RT, et al. Combined coronary angiography and myocardial perfusion by

Cite this article as: Branch KR, Haley RD, Bittencourt MS, Patel AR, Hulten E, Blankstein R. Myocardial computed tomography perfusion. Cardiovasc Diagn Ther 2017;7(5):452462. doi: 10.21037/cdt.2017.06.11 computed tomography in the identification of flowlimiting stenosis - The CORE320 study: An integrated analysis of CT coronary angiography and myocardial perfusion. J Cardiovasc Comput Tomogr 2015;9:438-45.

36. Chen MY, Rochitte CE, Arbab-Zadeh A, et al. Prognostic value of combined CT angiography and myocardial perfusion imaging versus invasive coronary angiography and nuclear stress perfusion imaging in the prediction of major adverse cardiovascular events: The CORE320 Multicenter Study. Radiology 2017;284:55-65. 